\title{
Tuning and Emulation of Mechanical Characteristics - Tunable Mounts and a Mechanical Hardware-in-the-Loop Approach for More Efficient Research and Testing
}

\author{
Jonathan Millitzer ${ }^{(凶)}$, Jan Hansmann, Giovanni Lapiccirella, Christoph Tamm, \\ and Sven Herold
}

Fraunhofer Institute for Structural Durability and System Reliability LBF, 64289 Darmstadt, Germany

Jonathan.Millitzer@lbf.fraunhofer.de

\begin{abstract}
Numerical simulations offer a wide range of benefits, therefore they are widely used in research and development. One of the biggest benefits is the possibility of automated parameter variation. This allow testing different scenarios in a very short period of time. Nevertheless, physical experiments in the laboratory or on a test rig are still necessary and will still be necessary in the future. The physical experiments offer benefits e.g. for very complex and/or nonlinear systems and are needed for the validation of numerical models.

Fraunhofer LBF has developed hardware solutions to bring the benefit of rapid and automated parameter variation to experimental environments. These solutions allow the tuning and emulation of the mechanical properties, like stiffness, damping and eigenfrequencies of structures.

The work presents two approaches: First a stiffness tunable mount, which has been used in laboratory tests in the field of semi-active load path redistribution. It allowed the researcher to test the semi-active system under different mechanical boundary conditions in a short period of time. Second, a mechanical Hardware-inthe-loop (mHIL) approach for the NVH development of vehicles components is presented. Here a mHIL-system is used to emulate the mechanical characteristics of a vehicle's body in white in a wide frequency range. This allows the experimental $\mathrm{NVH}$ optimization of vehicle components under realistic boundary conditions, without actually needing a (prototype) body in white.
\end{abstract}

Keywords: Uncertainty · Smart dynamic testing · Tunable stiffness · Mechanical Hardware-in-the-loop

\section{Introduction}

\subsection{Uncertainties in Early Phases of the Product Development}

Uncertainty is considered a potential deficiency in any development phase of a technical system that has arisen due to a lack of information and/or knowledge. The behavior of a

(C) The Author(s) 2021

P. F. Pelz and P. Groche (Eds.): ICUME 2021, LNME, pp. 129-144, 2021.

https://doi.org/10.1007/978-3-030-77256-7_12 
mechanical system is not deterministic due to uncertainty in the system and its environment; i.e. the system behavior cannot be clearly determined [1]. Uncertainty Quantification (UQ) deals with the identification, quantification and reduction of uncertainty in models, experiments and their effects on selected targets of a technical system [2]. Using UQ errors are usually classified as being either random error (precision) or systematic error (bias). There are three kinds of uncertainties. Parameter uncertainty describes an uncertainty associated with the parameters of a numerical model. An error describes a recognizable deficit in the numerical simulation of a system. Model uncertainty describes the accuracy with which a numerical model depicts reality.

In many studies, the variations in the properties of a technical system are explored according to the procedure of the Uncertainty Quantification. Research focuses on the optimization of a technical system taking parameter uncertainty into account, and the determination of variations in system behavior due to parameter uncertainty in the system properties [3]. Variations in geometric, mechanical, electrical and material properties such as the length and thickness of a beam, the fuselage length and width of an aircraft, the mass and the damping coefficient of a vehicle body etc. are described either with intervals or with distribution functions such as normal and gamma distributions. Probabilistic simulation methods such as Monte-Carlo-Simulations are frequently used to determine the influences of parameter uncertainty on a system property. In most studies, the intervals and distribution functions used to describe the variations in system properties and parameters are based on the assumptions of the respective authors.

The numerical models of the investigated systems are often analytically well known, which allows a comparison between a system optimization with probabilistic and nonprobabilistic simulation methods. However, the validation of these models with experimental data is usually highly time-consuming or even impossible and a criterion for adequate and sufficient prediction is not defined or proposed. When it comes to experimental validation of numerical models for vibroacoustic applications especially two tasks within the experiment are often time-consuming:

1) The variation of mechanical characteristics like stiffness, damping or elastomerlike characteristics. Often different parts (e.g. rubber mounts with different stiffness and loss-factor) are needed to realize different characteristics and they need to be exchanged in the test setup.

2) The realization of adequate mechanical boundary conditions in case single components or subsystems are investigated. Therefore, often auxiliary constructions have to be designed and manufactured. E.g. auxiliary constructions are used to realize a desired dynamic stiffness as boundary conditions e.g. for active vibration reduction systems in a marine [4] and an automotive application [5].

Smart dynamic testing is an approach that can be used to make these experimental tests more efficient.

\subsection{Smart Dynamic Testing}

The basic idea behind smart dynamic testing is to make physical experiments in research and development as straightforward as possible for the researcher. This means the focus 
should be on the device under test (DUT) and the respective research objective. Additional efforts, e.g. auxiliary constructions as mechanical boundary conditions or the exchange of parts of the test setup to realize different configurations should be minimized. To make dynamic tests smarter and reduce time-consuming tasks there are two main approaches:

Rapid Parameter Variation of Mechanical Characteristics. Whether it comes to the mechanical boundary conditions of the DUT or mechanical characteristics like stiffness or damping values from parts of the DUT it is often beneficial if there is the opportunity to do a parameter variation on these values during experiments. Therefore, tools like tunable mounts, tunable vibration absorbers (TVA) or the later presented mechanical Hardware-in-the-loop system can be used. A brief overview is given in $[6,7]$ gives an application example of tunable mounts for laboratory tests in the field of uncertainty research.

Active Emulation of Mechanical Boundary Conditions. Typically, subsystems of a vehicle, e.g. suspension systems or drive trains, are developed and tested by suppliers. In the vehicle, the body in white defines the mechanical boundary conditions of these subsystems. Especially in early stages of the development process, the body in white of the vehicle is often not available. Nevertheless, the correct boundary conditions are crucial for the vibroacoustic development [8] as well as for durability testing [9]. Instead of designing and building auxiliary constructions with a desired dynamic characteristic, these characteristic can be emulated by an active system. This emulation is often referred to (mechanical) Hardware-in-the-loop testing. Different application examples can be found in [10-16].

Figure 1 shows an exemplary test setup for noise, vibration and harshness (NVH) investigations on an electric drivetrain, which implements these two approaches. The drivetrain with its subframe (1) and the rubber mounts is the DUT.

Goal of this setup is to test the DUT under mechanical boundary conditions close to the actual installation situation in the vehicle in order to reduce uncertainties in the NVH development process. From a vibroacoustic point of view, the installation setup is characterized by its mechanical boundary conditions i.e. the dynamic stiffness of the body in white at the mounting points (reference characteristic). The shown setup allows the active emulation of this reference characteristic through four mechanical Hardware-in-the-loop interfaces (4).

Further, a tunable vibration absorber (3) is shown as an example for rapid parameter variation in physical test setups. It allows to test different vibration absorber configurations (tuning frequency and damping) without actually exchanging the vibration absorber in the test setup. Nevertheless the focus of this paper is the mHIL-system for the emulation of the mechanical boundary conditions.

Focusing on the reduction of uncertainties during early stage development and hence the correct emulation of the boundary conditions, the requirements depend on the actual research objective. Core requirements are the number of mechanical degrees of freedom (DOF) and the frequency range in which the emulated characteristics should be close to the reference characteristics. 
(2)

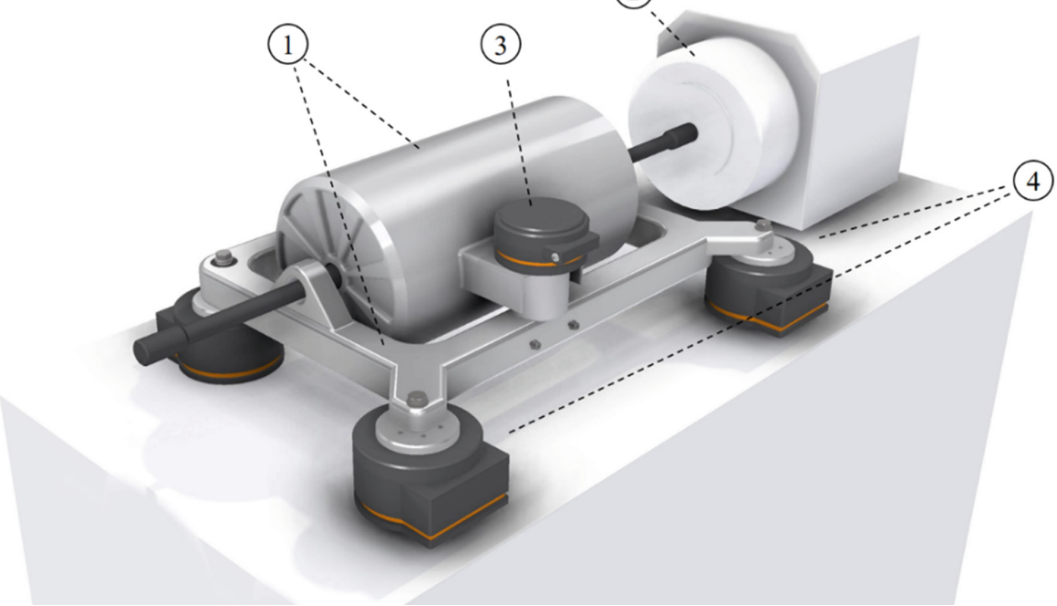

Fig. 1. Schematic test setup for NVH investigations on an electric drivetrain with: (1) drivetrain with subframe as DUT, (2) driven machine, (3) tunable vibration absorber as an example for rapid parameter variation and (4) four mHIL-interfaces for active emulation of the DUTs mechanical boundary conditions.

In this paper, the results of a 1-DOF active emulation of the dynamic stiffness are shown and discussed. The requirements were chosen according to typical issues in the field of automotive NVH development. The dynamic stiffness range reaches from $500 \mathrm{~N} / \mathrm{mm}$ (rubber mount) up to $10.000 \mathrm{~N} / \mathrm{mm}$ (body in white) and the considered frequency range reaches from $0 \mathrm{~Hz}$ to $1 \mathrm{kHz}$.

\section{Active Dynamic Stiffness Emulation by the Mechanical Hardware-in-the-Loop Approach}

\subsection{The Mechanical Hardware-in-the-Loop (mHIL) System}

Figure 2 gives an overview of the main components of the mHIL-system used to emulate the dynamic stiffness which defines the mechanical boundary condition for the DUT. The dynamic stiffness is defined by the user in a numerical simulation model, e.g. a finite element model. This numerical simulation model is converted into a numerical realtime capable model, the target model. Based on this target model the mHIL-interface is controlled using an adaptive controller which minimizes the difference between the target behavior and the actual behavior measured between the mHIL-interface and the DUT.

Numerical target models of the full system or of individual components of the system are set up with common analytical or numerical tools. Usually the Finite Element method in combination with suitable model order reduction methods are used in simulation [17]. In the dynamic testing environment, the simulation models have to be solved in real-time, i.e. the simulation has to meet requirements regarding timeliness, 


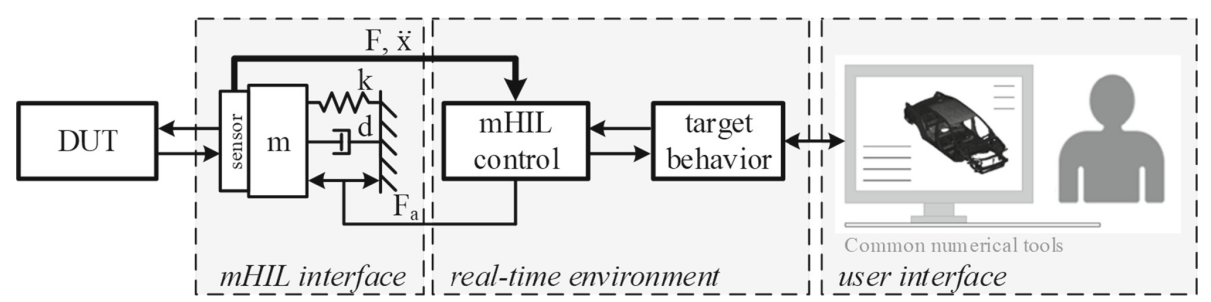

Fig. 2. Overview of the mHIL-system.

simultaneousness and responsiveness. The mandatory model properties are dependent on the specific characteristics of the mHIL-system, e.g. frequency range, computational power of the real-time simulator.

The mHIL-interface demonstrator was designed to allow 1-DOF dynamic stiffness emulation for typical scenarios in automotive testing. [6] shows an example where this interface was used for the characterization of automotive shock absorbers for different installation scenarios. Figure 3 shows the basic topology of the interface, which is mounted to a surface and can be connected to the DUT at its moved mass.
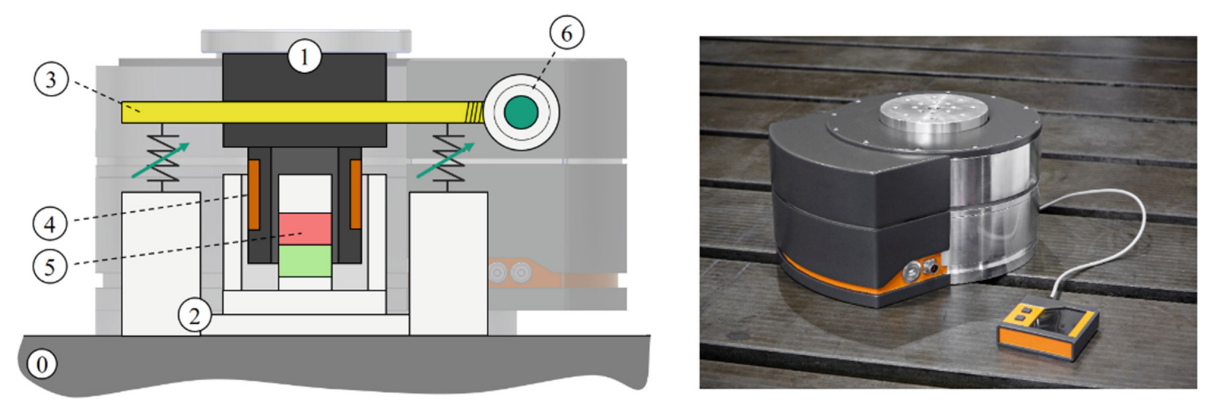

Fig. 3. Topology of the mHIL-interface with: The mounting surface (0), the moved mass (1), the housing (2), the tunable spring (3), the voice-coil actuators coil (4) and magnet (5) and the worm gear and electric motor for stiffness tuning (6) (left). Picture of the interface (right).

When it comes to the actual mechanical design of the mHIL-interface it is beneficial to have a low moved mass, a small installation space and no mechanical resonances in the frequency range in which the target behavior should be emulated. The focus in the presented work was on the emulation capability of the whole mHIL-system, the requirements "installation space" and "low moved mass" of the interface had no priority for the presented design and are to be further optimized with respect to a distinct application.

Compared to [8] a voice coil actuator (VCA) is used instead of a piezo-actuator. Goals were to keep the costs low, have the possibility to use off the shelf components (VCA and HiFi amplifiers), and have a mechanical robust design for the use in the test field. 
The semi-active, tunable spring in parallel to the actuator is used to keep the force requirements of the actuator low, especially for applications with higher static loads. The tunable spring was realized using the mechanism presented in [18]. Figure 4 shows the basic principle, which is to change to free length $\varphi$ of a ring segment to change its stiffness. This stiffness change is realized by the rotation of two structures relative to each other. In the interface, this rotation is realized by an electric motor using a planetary and a worm gear in serial.

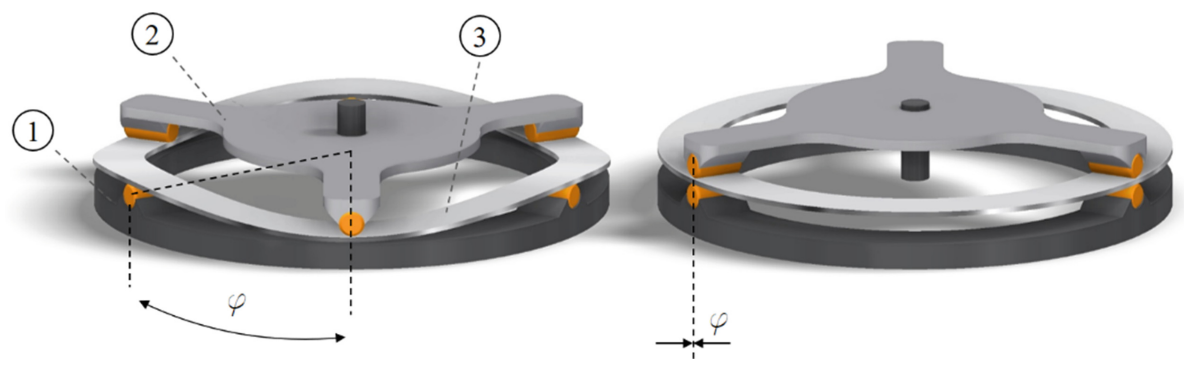

Fig. 4. Principle of semi-active stiffness tuning, with the first structure (1), the second structure (2), the spring element (3), and the tuning angle $\varphi$. Lowest stiffness setting (shown in deflected condition) (left). Highest stiffness setting (right).

The tunable stiffness was designed to have a minimum stiffness of $400 \mathrm{~N} / \mathrm{mm}$. This leads to an inner diameter of $160 \mathrm{~mm}$ and an outer diameter of $200 \mathrm{~mm}$ for the spring element, which was made out of high strength spring steel. The maximum stiffness is mainly defined through the compliance of the surrounding components like the interfaces housing.

The chosen VCA (Type "BEI Kimco, LA30-43-000A") has a peak force of 445 N, a continuous force of $185 \mathrm{~N}$ and a stroke of $\pm 12 \mathrm{~mm}$. The weight of the coil is $726 \mathrm{~g}$ and the weight of the magnet assembly is $1.9 \mathrm{~kg}$. To keep the moved mass of the interface low the magnet is attached to the housing and the coil is part of the moved mass. The total mass of the interface is about $20 \mathrm{~kg}$, whereas the moved mass is between $4.3 \mathrm{~kg}$ (highest stiffness setting) and $4.8 \mathrm{~kg}$ (lowest stiffness setting).

For a first mechanical characterization, the interface was excited with a white noise actuator current and the acceleration on the moved mass was measured. Figure 5 shows the magnitude response of the $\mathrm{H} 1$ transfer function estimate between the voice coil's current and the acceleration at the moved mass of mHIL-interface for different stiffness settings.

For the lowest stiffness setting the resonance of the interfaces moved mass is at $49.8 \mathrm{~Hz}$ and for the highest stiffness setting the resonance can be estimated around $590 \mathrm{~Hz}$. Considering the mHIL-interface as a simplified spring-mass-damper system this corresponds to a tuning range from $470 \mathrm{~N} / \mathrm{mm}$ to $59.100 \mathrm{~N} / \mathrm{mm}$ for the stiffness element.

Further, there are effects which are considered to be caused by structural resonances of components of the interface itself, e.g. at $290 \mathrm{~Hz}$, between $550 \mathrm{~Hz}$ and $730 \mathrm{~Hz}$ and 

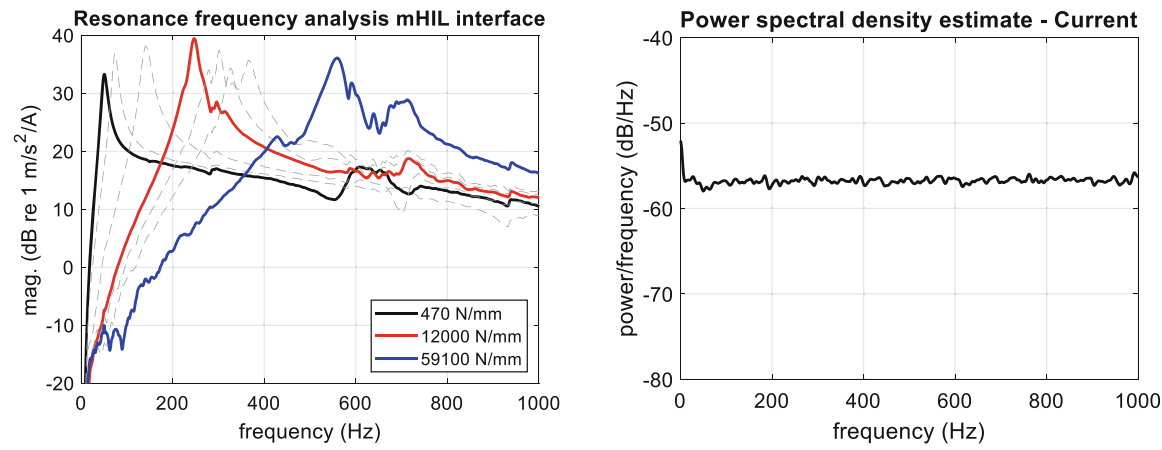

Fig. 5. Characterization results of the uncontrolled mHIL-interface for different settings of the tunable stiffness (left). Bandlimited white noise excitation of the voice coil's current used for characterization (right).

at $935 \mathrm{~Hz}$. The cause of these effects is subject of ongoing research with the goal to eliminate these parasitic effects in a next iteration of the interface design.

An adaptive controller is used to adjust the mHIL-interface's movement based on the measured reaction force between the DUT and the mHIL-interface's mounting surface. Preferring an adaptive controller, i.e. a real-time estimator [19] over a fixedparameter control approach, is motivated by two major facts: Firstly, the dynamics of the control path might be hard to model as they incorporate the conflated dynamics of the DUT, the mHIL-interface and the target behavior and thus an experimental modelling approach is highly advisable. Secondly, due to its iterative adaptation process the adaptive controller is able to minimize the controller's objective function even if slight deviations within the system occur. For an overview on possible mHIL control approaches, the reader is kindly referred to $[20,21]$.

\subsection{Test Setup and Test Cases}

Experimental investigations were carried out and focused on a preliminary study assessing synthetic test cases based on the emulation of the principle dynamic stiffness behavior present in an exemplary automotive application (c.f. Sect. 2.3). Envisioning a mechanical HIL test scenario, a substructure of a passenger car's chassis will be connected to the mHIL-interface, which in turn emulates the mechanical boundary condition the substructure would have experienced when installed into the car's chassis. Figure 6 shows the experimental test setup. The mHIL-interface demonstrator is mounted on a rigid supporting structure. An electrodynamic shaker is connected to the mHIL-interface through an impedance measurement head. The electrodynamic shaker introduces a broadband colored noise mechanical excitation into the interface, which in turn emulates the desired mechanical boundary condition by measuring the reaction force at the shaker's mounting position and by controlling the interface's movement (i.e. acceleration). Figure 6 also illustrates the test setup including the digital signal processing chain. The mHILinterface is represented by the lumped parameter model including the tunable stiffness element $k_{i}$, a presumed viscous damping $c_{i}$, and the mHIL-interface's moved mass $m_{i}$. 
In addition, the mHIL-interface incorporates a force actuator $\mathrm{F}_{\mathrm{a}}$. The mHIL-interface is connected to a lumped parameter model of the electrodynamic shaker given by the shaker's mass $\mathrm{m}_{\mathrm{s}}$, it's mechanical stiffness $\mathrm{k}_{\mathrm{s}}$ and an assumed viscous damper $\mathrm{c}_{\mathrm{s}}$. The excitation signal is used for both, the introduction of the excitation shaker force $F_{s}$ as well as the generation of the control signal $F_{a}$ computed by an adaptive feedforward controller.
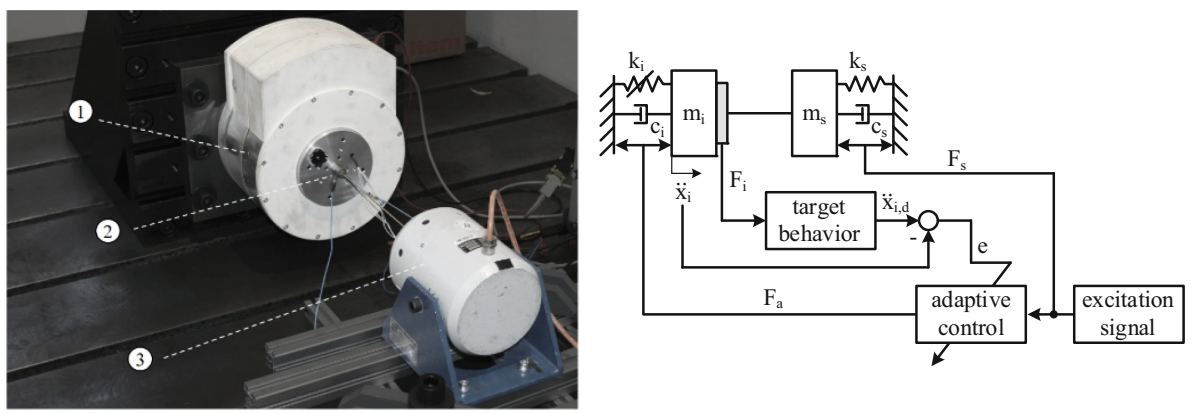

Fig. 6. Mechanical HIL test setup comprising the mHIL-interface demonstrator (1) and a primary mechanical excitation by means of an additional electrodynamic shaker (3). An impedance measurement head (2) captures interface forces and accelerations (left). Schematic illustration of the test setup including the adaptive controller and the numerical target model behavior (right).

Once an excitation force $\mathrm{F}_{\mathrm{S}}$ has been introduced into the test rig by means of the electrodynamic shaker, the reaction force $F_{i}$ is fed into a numerical model of the target mechanical behavior. The desired interface acceleration $\ddot{x}_{i, d}$ is derived from the measured interface force $\mathrm{F}_{\mathrm{i}}$ and the numerical model of the target behavior. It is then compared to the actual, measured interface acceleration $\ddot{x}_{i}$. The deviation between the desired acceleration $\ddot{x}_{i, d}$ and the measured acceleration $\ddot{x}_{i}$ serves the computation of an error signal e. The adaptive control algorithm's objective is to minimize the Least-MeanSquares error signal by means of an overlap-save frequency-domain Newton's algorithm [22]. Making use of feedforward topology, the controller is unconditional stable ensuring a bounded-input-bounded-output stability.

Table 1 shows the test cases that have been carried out with this setup. The motivation behind the selected test cases was to have a first proof for the mHIL-system to emulate different dynamic systems. Within this, the ability to vary the (static) stiffness, insert resonance effects and vary their damping are evaluated.

\subsection{Test Results}

Due to their high comparability, detailed experimental results are presented for the exemplary test case B-2 within the beginning of this section. A final assessment of all test cases is given at the end of this section (Fig. 10 and Fig. 11).

Experimental investigations are carried out for a stochastic excitation signal whereas bandlimited red noise with a maximum bandwidth of $1 \mathrm{kHz}$ is introduced by the electrodynamic shaker. Figure 7 (left) shows the frequency response function of the numerical 
Table 1. Synthetic test cases.

\begin{tabular}{l|l|l|l|l}
\hline Test case & $\begin{array}{l}\text { Quasistatic } \\
\text { stiffness }(\mathrm{N} / \mathrm{mm})\end{array}$ & $\begin{array}{l}1^{\text {st }} \text { resonance } \\
\text { frequency }(\mathrm{Hz})\end{array}$ & $\begin{array}{l}\text { Modal damping } \\
(\%)\end{array}$ & $\begin{array}{l}2^{\text {nd }} \text { resonance } \\
\text { frequency }(\mathrm{Hz})\end{array}$ \\
\hline A-1 to A-3 & 500 to 10,000 & - & - & - \\
\hline B-1 to B-3 & 2,250 & 300 & 1 to 100 & - \\
\hline C-1 to C-3 & 2,250 & 100 to 500 & 10 & - \\
\hline D-1 to D-3 & 2,250 & 190 & Approx. 10 & 327 to 700 \\
\hline
\end{tabular}

target behavior for the test case B-2. The frequency response function shows a single resonance frequency at $300 \mathrm{~Hz}$ with a modal damping of $10 \%$. The computed desired acceleration $\ddot{\mathrm{x}}_{\mathrm{i}, \mathrm{d}}$ and the measured acceleration signal $\ddot{\mathrm{x}}_{\mathrm{i}}$ serve the calculation of the error signal e.
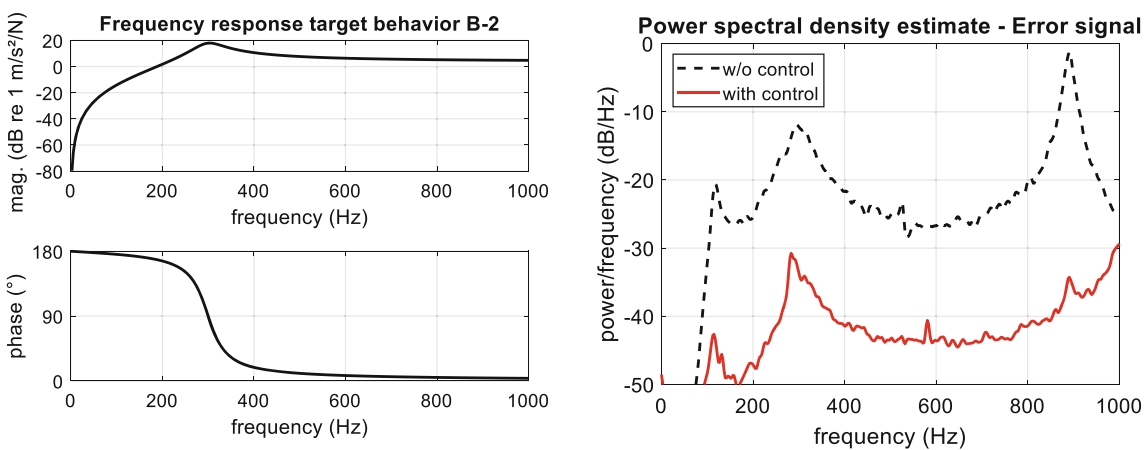

Fig. 7. Frequency response function of the numerical target behavior B-2 (left) and power spectral density estimate of the deviation between the actual mHIL-interface acceleration and the desired acceleration derived from the numerical model of the target behavior (right).

Figure 7 (right) illustrates a power spectral density (PSD) estimate of the error signal e. Deviations between the target and the desired acceleration with respect to the bandlimited red noise excitation are mainly observed at the three distinct resonance frequencies of the test setup. Two of the resonance frequencies originate from the mechanical test setup itself. The first resonance frequency at approx. $160 \mathrm{~Hz}$ originates from the mechanical resonance frequency of the mHIL-interfaces tunable stiffness $k_{i}$ and the attached masses of the mHIL-interface $m_{i}$ and the electrodynamic shaker $m_{s}$, respectively. The highest resonance frequency at approx. $890 \mathrm{~Hz}$ is observed due to a parasitic effect within the test setup and most likely results from a leak in stiffness of the used connection rod between the electrodynamic shaker and the mHIL-interface. This connection will be improved in further investigations. In addition, the third resonance frequency at $300 \mathrm{~Hz}$ within the measured PSD of the error signal e is caused by the numerical model of the target behavior. Hence, it should be noted that the adaptive controller has to deal with 
a control system whose dynamic properties originate from both the actual physical test rig setup as well as the dynamics given by the numerical model of the target behavior.

Once the adaptive controller is enabled and a steady state within the iterative controller adaptation process has been reached, the PSD of the error signal e is significantly lowered. A broadband reduction of -20 to $-40 \mathrm{~dB}$ can be observed within the whole frequency band up to the target operational frequency of $1 \mathrm{kHz}$.

Reducing the deviation between the actually measured interface acceleration $\ddot{x}_{i}$ and the desired interface acceleration $\ddot{x}_{i, d}$ depicts that the mHIL-interface's mechanical behavior follows the target behavior given by the implemented numerical model. This also gets obvious considering the target and the measured acceleration signals in time domain. Figure 8 shows an exemplary section of the time series of the initial state (left). Disabling the control signal of the mHIL-interface, a significant deviation between the target acceleration and the actual measured interface acceleration can be observed. Once the adaptive controller is enabled only small deviations occur between the target behavior and the actual measured interface acceleration (c.f. Fig. 8, right).
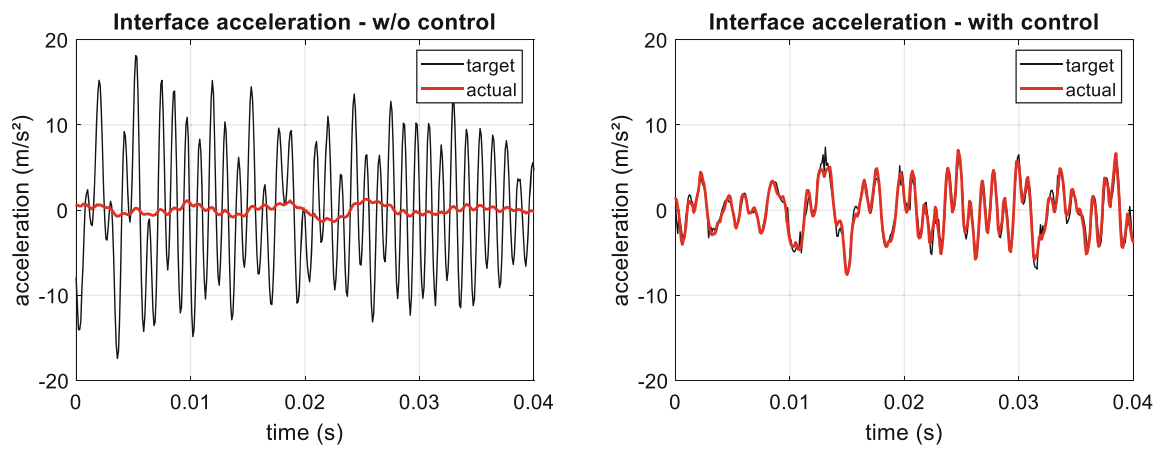

Fig. 8. Time series of the desired mHIL-interface's acceleration $\ddot{x}_{i, d}$ computed by the numerical model of the target behavior and the actual measured interface acceleration $\ddot{x}_{i}$.

Figure 9 shows the PSD estimates for both the mHIL-interface's acceleration $\ddot{x}_{i}$ (left) and the measured reaction force $\mathrm{F}_{\mathrm{i}}$ (right). Again, significant changes can be observed, once the mHIL-interface's adaptive controller has been enabled and has reached steady state. For the uncontrolled case, the mHIL-interface's acceleration PSD estimate is mainly dominated by the resonance frequencies at approx. $160 \mathrm{~Hz}$ and at approx. $890 \mathrm{~Hz}$ resulting from the physical test setup. This behavior significantly changes once the mHIL-interface's controller is enabled. Here, the measured interface's acceleration PSD estimate is dominated by the resonance frequency at $300 \mathrm{~Hz}$ originating from the numerical model of the target behavior B-2. Changes within the PSD estimate can also be observed for the measured reaction force's PSD estimate whereas changes mainly occur within the frequency range above $100 \mathrm{~Hz}$. A considerable reduction of the reaction force $\mathrm{F}_{\mathrm{i}}$ for the controlled case is mainly observed at the parasitic resonance frequency at $890 \mathrm{~Hz}$.

In order to assess the conflated behavior that results from both a change for the mHIL-interface's acceleration $\ddot{x}_{i}$ as well as the interface's reaction force $F_{i}$, the dynamic 

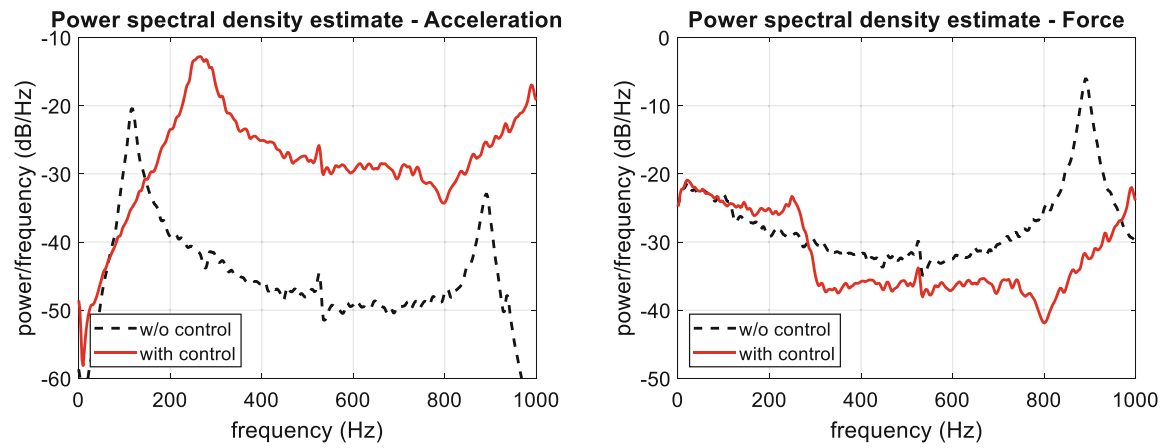

Fig. 9. Power spectral density estimate of the measured mHIL-interface's acceleration $\ddot{x}_{i}$ (left) and reaction force $\mathrm{F}_{\mathrm{i}}$ (right). The black dashed line depicts the uncontrolled case (i.e. no control signal is fed to the mHIL-interface's actuator). The solid red line illustrates the measured steady state behavior.

stiffness frequency response has been calculated by means of both measured signals. Assuming the measurement noise to be uncorrelated to the input signals, the $\mathrm{H} 1$ method has been used for deriving the dynamic stiffness frequency response functions. Figure 10 and Fig. 11 show the estimated frequency response functions for the test cases A and B as well as for the test cases $\mathrm{C}$ and $\mathrm{D}$, respectively. Due to a leak in coherence within the frequency range below $70 \mathrm{~Hz}$, which is caused by an impropriate signal-to-quantization noise ratio of the analog-to-digital-converter for the acceleration measurement channel, the $\mathrm{H} 1$ transfer function estimate in the lower frequency range is shown only for the sake of completeness and has to be considered untrustworthy.

The test cases A-1 to A-3 (c.f. Fig. 10) show a varying quality in emulating the mechanical characteristics given by the numerical target behavior model. For the lowest stiffness setting (c.f. test case A-1, Table 1) a good emulation is achieved within the frequency range from approx. $100 \mathrm{~Hz}$ to $420 \mathrm{~Hz}$. Deviations mainly occur in the higher frequency range above $420 \mathrm{~Hz}$. For both test cases A-2 and A-3, the controlled mHILinterface shows a sufficient performance, except for the frequency range at approx. $890 \mathrm{~Hz}$. For the test cases B-1 to B-3, Fig. 10 illustrates the good performance of the controlled mHIL-interface up to the target frequency range of $1 \mathrm{kHz}$. Slight deviations occur for the test case B-1. Here, the amplitude response shows an error of a factor of five within the sharp resonance frequency (modal damping of only 1\%) implemented by the numerical model of the target behavior B-1.

Figure 11 shows the exemplary results obtained for the test cases $\mathrm{C}$ and D (c.f. Table 1). The mHIL system shows a good performance for the test cases C. Hence, the controlled interface is able to emulate a shift in the resonance frequency for the considered test cases. Again, small deviations are observed in the frequency range at approx. $890 \mathrm{~Hz}$. Introducing a second resonance frequency in the test cases D, the mHILinterface is also able to emulate the desired mechanical behavior based on the numerical target model with slight restrictions. For the test case C-3 and D-2, slight deviations occur in the frequency range at approx. $890 \mathrm{~Hz}$ due to the aforementioned reasons. 

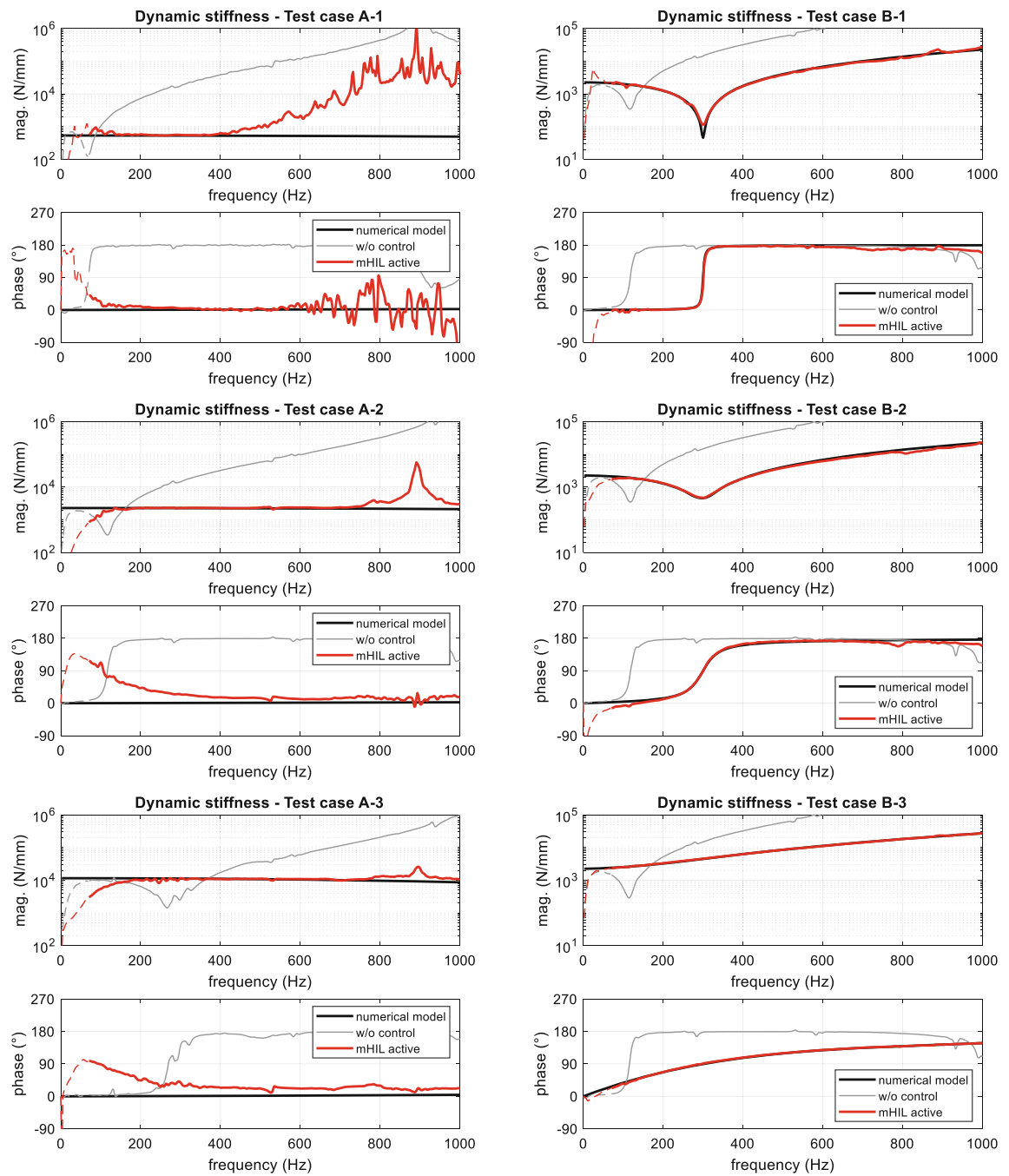

Fig. 10. Conflated behavior of the controlled mHIL-interface for test cases A (left, top to bottom) and $\mathrm{B}$ (right, top to bottom) evaluated by means of a $\mathrm{H} 1$ transfer function estimate of the dynamic stiffness computed from the measured interface's acceleration $\ddot{x}_{i}$ and reaction force $F_{i}$.

\section{Discussion}

The emulation of an ideal dynamic stiffness, the emulation of a single resonance frequency with varying modal damping and resonance frequency, and the emulation of a multi-resonant mechanical behavior (c.f. Table 1) have been demonstrated successfully. To further illustrate this, Fig. 12 illustrates the intended operating range of the controlled mHIL-interface with the shown test cases. The validations of the quasistatic stiffness ranges above $10.000 \mathrm{~N} / \mathrm{mm}$ and the higher frequencies above $1 \mathrm{kHz}$ are subject to current research activities. 

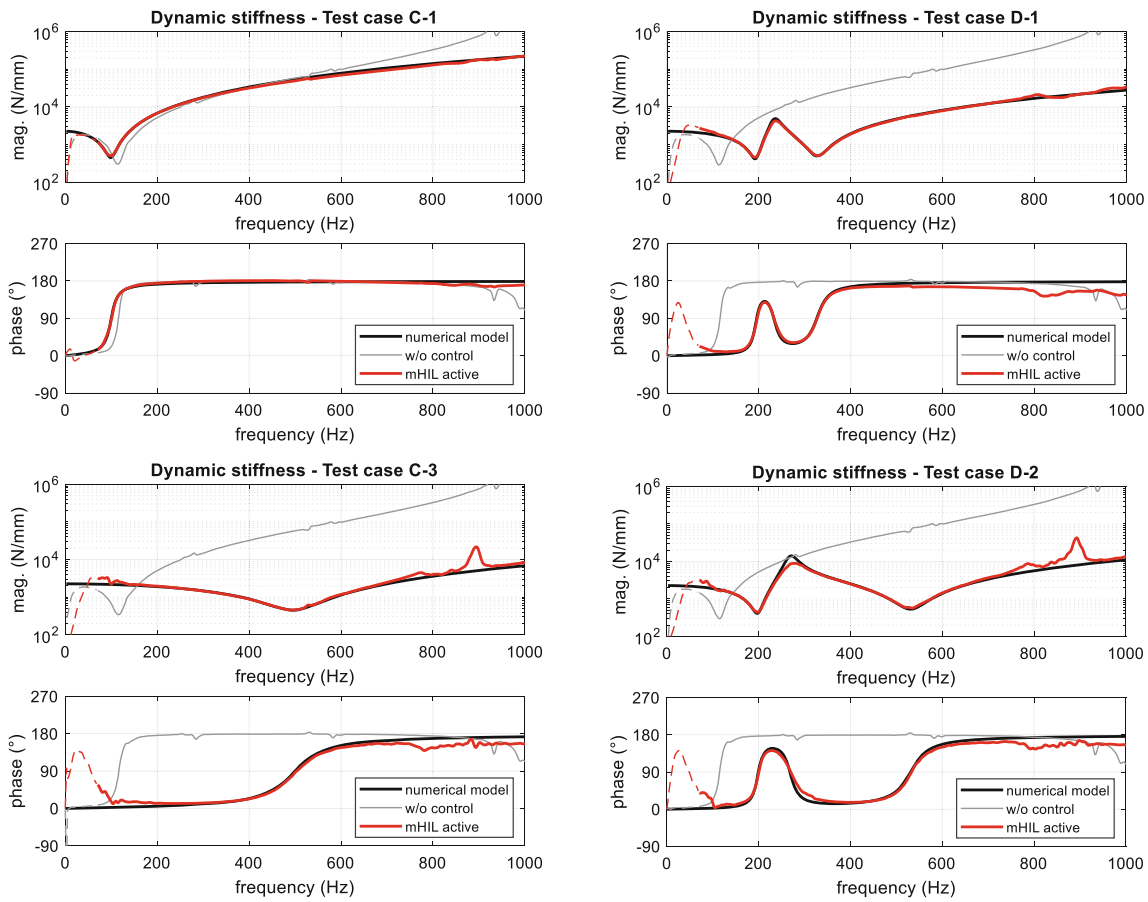

Fig. 11. Conflated behavior of the controlled mHIL-interface for exemplary test cases C (left) and $\mathrm{D}$ (right) evaluated by means of a $\mathrm{H} 1$ transfer function estimate of the dynamic stiffness computed from the measured interface's acceleration $\ddot{x}_{i}$ and reaction force $F_{i}$.

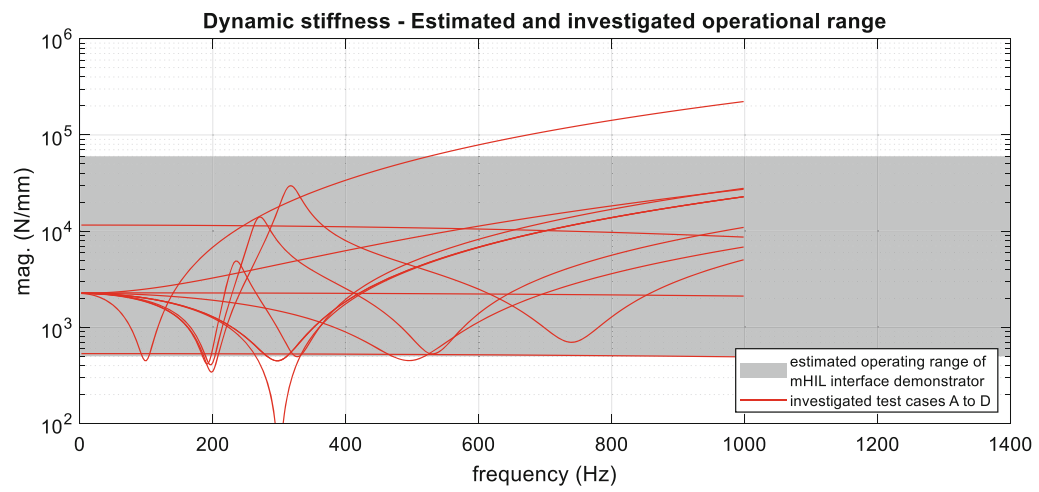

Fig. 12. Esitmated operational range (shaded area) and investigated operational range of the mHIL-interface demonstrator.

In the investigated operating range, limitations of the current setup mainly occur in the lower frequency range below $70 \mathrm{~Hz}$. Here, performance limitations are mainly due to an inappropriate data acquisition setup of the digital signal processing chain. Even though the utilized dSPACE DS2004 analog-to-digital conversion hardware offers a resolution 
of 16 bit, an effective resolution of only 12 bit has been observed taking into account quantization and measurement noise. Hence, the effective dynamic range of analogto-digital converter is approx. $72.2 \mathrm{~dB}$, which is already close to the overall dynamic range of approx. $50 \mathrm{~dB}$ of the measured acceleration signal (c.f. Fig. 9, PSD estimate of acceleration signal). An improvement of the performance of the controlled mHILinterface can thus be achieved by either reducing measurement noise, by introducing a sensor fusion technique for the lower and higher frequency range, or by increasing the dynamic range (i.e. increasing the effective bit resolution) of the analog-to-digital converter incorporating an alternative digital signal processing hardware.

The limited performance for the emulation of an ideal stiffness element (i.e. test cases A, c.f. Table 1 and Fig. 10) might be caused by the phase response of the numerical model for the target behavior. Introducing a second order derivative behavior, the phase response shows a $+180^{\circ}$ phase advantage whenever the desired mHIL-interface's acceleration is computed. Taking into account the minimum reaction time of the conflated secondary path behavior, the Wiener optimal solution of the adaptive feedforward control problem might thus be non-causal in the higher frequency range. This issue might be addressed by further reducing the overall reaction time of the conflated secondary path behavior, which requires an increase of the digital signal processing frequency fairly above the so far utilized sampling frequency of $10 \mathrm{kHz}$, the application of fast-conversion analog-to-digital and digital-to-analog converters as well as tailored anti-aliasing and reconstruction filters respectively. Considering the hardware effort required to achieve an appropriate control performance for the synthetic test cases incorporating an ideal stiffness element (i.e. test cases A), an end users survey has to reveal the importance of these test cases. Physical equivalent test cases are deemed to be occasional and found e.g. in the development of small optical instruments or within the semiconductor, MEMS, or sensor industry.

\section{Conclusion}

The test results obtained within the experimental proof of concept for the controlled mHIL-interface demonstrator depict a quite promising result regarding the application of the mHIL technology in automotive NVH development, especially if uncertainties should be considered. In accordance to the increased interest in uncertainty quantification, there exists a demand for appropriate experimental test equipment within structural dynamics. As uncertainties in structural dynamics often arise from the installation conditions of mechanical substructures and components, future test equipment must be able to emulate the mechanical boundary condition (i.e. installation condition) with high precision and bandwidth. Furthermore, the test equipment must be able to change its mechanical behavior ensuring ease of use.

Within this paper, a mHIL-system demonstrator was experimentally validated, demonstrating the highly automatable capability to change a mechanical boundary condition. It is based on both the application of a tunable mechanical stiffness element as well as the incorporation of a numerical model of a target mechanical behavior in an adaptive control loop. Investigations for different synthetic test cases illustrate the capabilities of emulating mechanical properties up to a frequency of $1 \mathrm{kHz}$ and thus enabling experimental probabilistic analyses within the field of uncertainty quantification. 
As a next step the mHIL-system demonstrator should be further developed towards industrial application, whereat a close cooperation with industrial partners is necessary.

Acknowledgements. Parts of the work have been funded by the Fraunhofer research project »Light Materials 4 Mobility LM4M « and the Fraunhofer technology transfer program »AHEAD « [23]. The financial support is gratefully acknowledge.

\section{References}

1. Oberkampf, W.L., Deland, S.M., Rutherford, B.M., Diegert, K.V., Alvin, K.F.: Error and uncertainty in modeling and simulation. Reliab. Eng. Syst. Saf. 75(3), 333-357 (2002)

2. Smith, R.C.: Uncertainty Quantification: Theory, Implementation, and Applications. Bd. 12. SIAM (2013)

3. Platz, R., Ondoua, S., Enss, G.C., Melz, T.: Approach to evaluate uncertainty in passive and active vibration reduction. In: Model Validation and Uncertainty Quantification, Volume 3: Proceedings of the 32nd IMAC, A Conference and Exposition on Structural Dynamics, pp 345-352. Springer International Publishing (2014)

4. Matthias, M., Friedmann, A., Koch, T., Drögemüller, T.: Active mounts for marine application; the BMBF research project "active aggregate mounts to reduce structure vibrations and structure-borne sound transmission. In: Proceedings of SPIE (2006)

5. Kraus, R.: Auslegung und Umsetzung eines Labordemonstrators zur aktiven Lagerung im Fahrwerksbereich eines Kraftfahrzeugs. Diplomarbeit, Fachhochschule Gießen-Friedberg (2010)

6. Hansmann, J., Millitzer, J., Rieß, S., Balzer, L.: Symbiose virtueller und experimenteller Methoden für effizienteres Testen und Entwickeln. In: Experten-Forum Powertrain: Simulation und Test 2019. Springer Vieweg, Deutschland, Wiesbaden (2020). ISBN: 978-3-658-28706-1

7. Gehb, C.M., Platz, R., Melz, T.: Two control strategies for semi-active load path redistribution in a load-bearing structure. Mech. Syst. Sig. Process. 118, 195-208 (2019). https://doi.org/ 10.1016/j.ymssp.2018.08.044

8. Mayer, D., Jungblut, T., Wolter, S., Millitzer, J.: Hardware-in-the-loop test environments for vibration control systems. In: NAFEMS Seminar Practical Aspects of Structural Dynamics, Deutschland, Wiesbaden (2015)

9. Landersheim, V., Möller, R., Dittmann, K.-J.: Ableitung Eines Vereinfachten Ersatzversuchs Für Pkw-Integralträger Mittels Numerischer Schädigungsbewertungen. In: Tagung des DVM Arbeitskreises Betriebsfestigkeit Potenziale im Zusammenspiel von Versuch und Berechnung in der Betriebsfestigkeit Volume: 43, Steyr, Österreich (2016)

10. Plummer, A.R.: Model-in-the-loop testing. Proc. Inst. Mech. Eng. Part I J. Syst. Control Eng. 220, 183-199 (2006)

11. Wolter, S., Franz, R., Jungblut, T., Möller, R., Bruder, T.: Aktive Anbindungsimpedanzen: Ein Anwendungsszenario echtzeitfähiger Lagermodelle. DVM-Workshop Elastormerbauteile, Weinheim (2011)

12. Facchinetti, A., Bruni, S.: Hardware-in-the-loop hybrid simulation of pantograph-catenary interaction. J. Sound Vib. 331(12), 2783-2797 (2012)

13. Atzrodt, H., Mayer, D., Koch, T., Vrbata, J.: Development of a multiaxial test environment for highly dynamically loaded mechatronic components. In: Symposium on Structural Durability SoSDiD 2017, Deutschland: Darmstadt, p. 15 (2017)

14. Bartl, A., Karamooz Mahdiabadi, M., Rixen, D.J.: Conception of a noise and vibration hardware-in-the-loop test bed. In: Proceedings of IMAC, a Conference and Exposition on Structural Dynamics (2017) 
15. Traphöner, P., Olma, S., Kohlstedt, A., Jäker, K.-P., Trächtler. A.: Universelle Entwicklungsund Prüfumgebung für mechatronische Fahrzeugachsen. Wissenschaftsforum Intelligente Technische Systeme (WInTeSys), pp. 11-12, May 2017 (2017)

16. Olma, S., Kohlstedt, A., Traphöner, P., Jäker, K.-P., Trächtler, A.: Observer-based nonlinear control strategies for Hardware-in-the-Loop simulations of multiaxial suspension test rigs. Mechatronics 50, 212-224 (2018)

17. Antoulas, A.C.: Approximation of large-scale dynamical systems. In: Advances in Design and Control. Society for Industrial \& Applied Mathematics, US (2005)

18. Hansmann, J., Kaal, W., Seipel, B., Melz, T.: Tuneable spring element for an adaptable vibration absorber. In: ATZ Worldwide, no. 03, pp. 36-39 (2012)

19. Åström, K.J., Wittenmark, B.: Adaptive control. Courier Corporation (2013)

20. Millitzer, J., Ranisch, C., Tamm, C., Bartolozzi, R., Melz, T.: An approach for the design of a mechanical Hardware-in-the-Loop interface. In: Symposium für Smarte Strukturen und Systeme 4SMARTS, Deutschland, Braunschweig (2017)

21. Millitzer, J., et al.: Recent developments in hardware-in-the-loop testing. In: Barthorpe, R. (ed.) Model Validation and Uncertainty Quantification, Volume 3. Conference Proceedings of the Society for Experimental Mechanics Series. Springer, Cham (2019). https://doi.org/10. 1007/978-3-319-74793-4_10

22. Elliott, S.J., Rafaely, B.: Frequency-domain adaptation of causal digital filters. IEEE Trans. Sig. Process. 48(5), 1354-1364 (2000). https://doi.org/10.1109/78.839982

23. Hybrid test solutions. https://www.hytestsolutions.com/. Accessed 30 Dec 2020

Open Access This chapter is licensed under the terms of the Creative Commons Attribution 4.0 International License (http://creativecommons.org/licenses/by/4.0/), which permits use, sharing, adaptation, distribution and reproduction in any medium or format, as long as you give appropriate credit to the original author(s) and the source, provide a link to the Creative Commons license and indicate if changes were made.

The images or other third party material in this chapter are included in the chapter's Creative Commons license, unless indicated otherwise in a credit line to the material. If material is not included in the chapter's Creative Commons license and your intended use is not permitted by statutory regulation or exceeds the permitted use, you will need to obtain permission directly from the copyright holder.

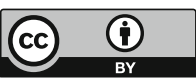

